TERRA. Revista de Desarrollo Local e-ISSN: 2386-9968

Número 6 (2020), 384-390

DOI 10.7203/terra.6.17003

IIDL - Instituto Interuniversitario de Desarrollo Local

\title{
Reseña. De lugar geográfico a destino turístico. Análisis, planificación y gestión de los procesos de cambio generados por el turismo
}

\author{
Juan Bautista Ferreres Bonfill \\ Departamento de Historia, Geografía y Arte. Gabinete de Estudios Turísticos (GETUR) \\ (Universitat Jaume I, Castellón, España) \\ ferreres@uji.es \\ https://orcid.org/0000-0002-6299-5029
}

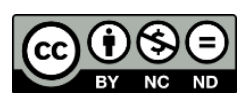

Esta obra se distribuye con la licencia Creative Commons Reconocimiento-NoComercial-SinObraDerivada 4.0 Internacional 


\section{SECCIÓN RESEÑAS}

\section{Reseña. De lugar geográfico a destino turístico. Análisis, planificación y gestión de los procesos de cambio generados por el turismo}

Resumen. La potencialidad del territorio para transformarse en destino turístico, es decir, de lugar geográfico a destino turístico, título de este libro, refleja la complejidad del fenómeno turístico, que se caracteriza por su transversalidad en el desarrollo, lo que posibilita ser abordado por diversas disciplinas. Es precisamente la Geografía, preocupada por los procesos de cambio que el turismo genera en los destinos y vulnerabilidad de estos frente a fenómenos que inducen cambios en su sistema, la que adquiere especial protagonismo, como disciplina de síntesis para estudiar la actividad turística desde una perspectiva integradora, cuyos resultados son de utilidad en la implementación de mecanismos de planificación y gestión. Este libro de actas trasmite los resultados de investigaciones en las expresadas temáticas, que son de plena actualidad, y que fueron debatidos en el XVI Coloquio de Geografía del Turismo, Ocio y Recreación, celebrado en la Universidad de León, en el mes de septiembre de 2018 y conformado por aportaciones de 82 autores.

Palabras clave: Movilidad, rutas y excursiones; sociedad local y desarrollo; normativa y planeamiento; gestión; impactos y sostenibilidad; nuevos recursos y modalidades turísticas.

Recibido: 06 de abril de 2020

Devuelto para revisión: -

Aceptado: 06 de abril de 2020

\section{Referencia / Citation:}

Ferreres, J. B. (2020). Reseña. De lugar geográfico a destino turístico. Análisis, planificación y gestión de los procesos de cambio generados por el turismo. TERRA. Revista de Desarrollo Local, (6), 384-390. DOI 10.7203/terra.6.17003 


\title{
Xosé Somoza Medina (Coord.)
}

\section{DE LUGAR GEOGRÁFICO A DESTINO TURÍSTICO. ANÁLISIS, PLANIFICACIÓN Y GESTIÓN DE LOS PROCESOS DE CAMBIO GENERADOS POR EL TURISMO}

\author{
Área de Publicaciones. Universidad de León, 2018. 478 páginas.
}

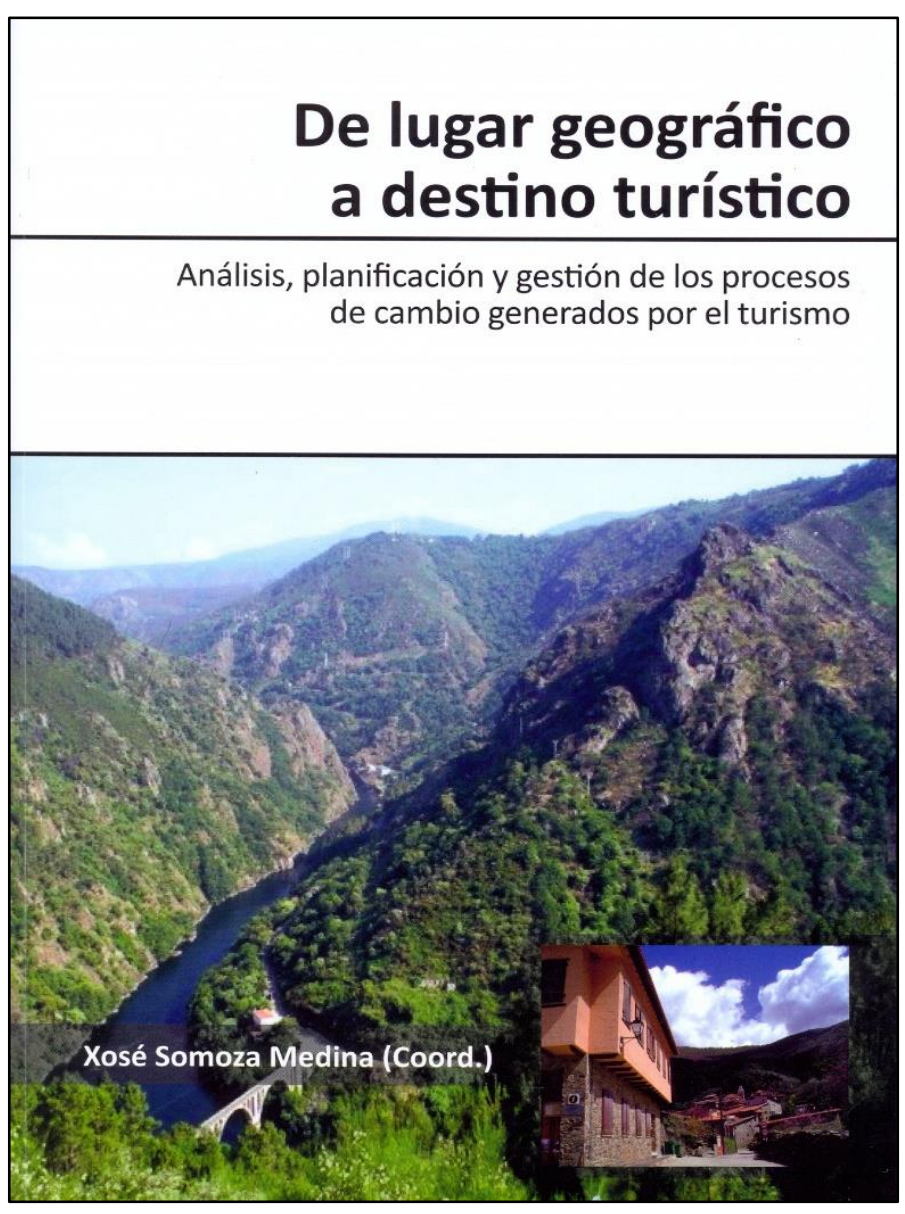

La potencialidad del territorio para transformarse en destino turístico, es decir, de lugar geográfico a destino turístico, título de este libro, refleja la complejidad del fenómeno turístico, que se caracteriza por su transversalidad en el desarrollo, lo que posibilita ser abordado por diversas disciplinas. Es precisamente la Geografía, preocupada por los procesos de cambio que el turismo genera en los destinos y vulnerabilidad de estos frente a fenómenos que inducen cambios en su sistema, la que adquiere especial protagonismo, como disciplina de síntesis para estudiar la actividad turística desde una perspectiva integradora, cuyos resultados son de utilidad en la implementación de mecanismos de planificación y gestión. Este libro de actas trasmite los resultados de investigaciones en las expresadas temáticas, que son de plena actualidad, y que fueron debatidos en el XVI Coloquio de Geografía del Turismo, Ocio y Recreación, celebrado en la Universidad de León, en el mes de septiembre de 2018 y conformado por aportaciones de 82 autores.

Es de reconocer el compromiso de la AGE, en el XVI Coloquio de Geografía del Turismo, Ocio y Recreación, con la memoria del Profesor Diego López Olivares, dedicándole este libro. Ha sido una gran pérdida para el mundo académico y en particular para la Geografía el Turismo, dejando un gran vacío para quienes hemos compartido su trayectoria personal y académica. La temática y contenido de esta obra se imbrica en la vertiente investigadora del profesor López Olivares, en la que la sistematización de los procesos turísticos desde una perspectiva integradora han marcado buena parte de su trayectoria. Esta es la razón de ser del grupo de investigación que dirigió, el Gabinete de Estudios Turísticos (GETUR), de la Universitat Jaume I de Castellón, en el que integró compañeras/os de distintas disciplinas y áreas de conocimiento, testimoniando su capacidad para entender el turismo desde una visión interdisciplinar que traduce la perspectiva de abordar la 
actividad turística a partir de los diferentes componentes que la integran, participan y explican su dinámica en el territorio.

El contenido de este libro de actas así lo refleja, estructurándose en seis grandes temas que se fundamentan en las ponencias del congreso: movilidad, rutas y excursiones; sociedad local y desarrollo; normativa y planeamiento; gestión; impactos y sostenibilidad; y nuevos recursos y modalidades. En este sentido, el primer capítulo, movilidad, rutas y excursiones, integra seis comunicaciones que reflejan resultados de investigaciones en torno a tres ejes. La vinculación del turismo con la movilidad es estrecha atendiendo a su vertiente territorial, y a ella se dedica el primer eje desde diversas escalas espaciales. Así, se analiza a escala local en los municipios de Catalunya a través de los Planes de Movilidad Urbana Sostenible, con resultados escasamente satisfactorios, al no existir una mención directa al turismo en este tipo de planificación. También desde la escala local se investiga la relación entre movilidad en destinos urbanos y la práctica excursionista, aplicada a una tipología turística como es el excursionismo cultural entre Madrid y Toledo. Pese a la política turística que se aplica en Toledo para incrementar la estancia media, son determinantes la mejora de la accesibilidad entre ambos destinos y la falta de implicación de los agentes turísticos no locales en seguir multiplicándose la oferta de viajes de un día, lo cual precisa una regulación y mayor cooperación privada-privada entre touroperadores y agentes turísticos de Madrid.

A escala internacional se aborda la estrecha relación entre las migraciones por motivos de ocio, vinculadas al lifestyle migration; y desde el lado de la oferta, las estrategias residenciales. Estas interrelaciones entre turista-residencial y turismo residencial se estudian en Marruecos ya que el desarrollo turístico ha generado nuevos espacios residenciales en los que se ha producido una apropiación material del patrimonio urbano para ser adaptado a las necesidades del turista-residente. En esta línea la homogeneización de la oferta turística provocada por la masificación y la pérdida del discurso basado en la cultura local se constata en San Pedro de Atacama (Chile), donde la segregación entre turistas y población local es clara. Incide en ello de manera decisiva el control que ejercen los operadores turísticos en focalizar la oferta solamente hacia rutas de paisaje y naturaleza. Sin dejar de lado el papel que juegan las rutas turísticas para articular una oferta diferenciada, se ha aplicado un análisis correlacional en torno a la Ruta Don Vasco, en México, para determinar la incidencia de la imagen turística en la gestión y conservación de los recursos patrimoniales y paisajísticos.

Bajo el título "Sociedad local y desarrollo" se articula el segundo capítulo, con seis comunicaciones, en las que el eje descansa en la relación entre la población local, la población residente y los espacios de destino turístico. Se constata en áreas supramunicipales rurales, en las que se han aplicado instrumentos de planificación turística territorial, que es débil la correspondencia entre la aplicación de modelos de gobernanza plena y la efectividad de las medidas implementadas en ello, ya que, pese a la participación social, se sigue priorizando el sector empresarial frente a la sociedad civil. Asimismo, se cuestiona en el litoral catalán la integración social en el tejido local de residentes calificados como trabajadores creativos en relación al resto de trabajadores. En lo que atañe a los destinos urbanos, en el centro histórico de Málaga la tensión se manifiesta entre el crecimiento turístico y la ciudadanía, ya que esta lo asocia con la construcción y lo inmobiliario, y los instrumentos de planificación no plantean soluciones. Sin embargo, en estas áreas, el fortalecimiento del asociacionismo y las redes de colaboración entre los agentes culturales, comerciales 
y turísticos fomentan el desarrollo y la sostenibilidad, como se ha demostrado en el Barrio de las Letras de Madrid y el Barrio Italia de Santiago de Chile. En el caso de los proyectos y experiencias en ecoturismo, el manejo grupal de los recursos naturales es viable siempre que exista una visión compartida, y se fomente la inversión en infraestructuras sostenibles y la inclusión social a nivel local, como lo atestiguan las experiencias estudiadas en el sureste mexicano y en áreas naturales de Colombia.

El tercer capítulo acoge seis comunicaciones relacionadas con la normativa, la planificación turística y con las políticas de ordenación del litoral, fundamental, dado el carácter territorial de la actividad turística. Así, desde la perspectiva normativa se reflexiona sobre la definición y el concepto del agroturismo que se desprende de la normativa autonómica. Si bien existe cierta unanimidad en clasificar el agroturismo con una tipología de turismo rural; sin embargo, adolece de una visión integral y no existe una definición única, lo cual repercute sobre su posicionamiento en el mercado. Desde la escala supramunicipal sobresale el estudio de la planificación turística al ser abordada en tres comunicaciones, en las que queda de manifiesto su complejidad y operatividad, resultando fundamental que los objetivos sean concretos, definidos desde el propio plan, acometidos a través de propuestas concretas y coherentes con el territorio; y dotados de un ente de gestión y con dotación presupuestaria, como se estudia en las comarcas del interior de Alicante. En el desarrollo de la planificación cobra especial relevancia la capacidad para generar dinámicas de producto y que incidan en la sostenibilidad del territorio, siendo este el principal reto en áreas de las cuencas mineras del carbón de Castilla y León, y en el turismo industrial en Asturias. Precisamente este vínculo entre el producto turístico y la sostenibilidad del desarrollo turístico es incorporado en la política de ordenación territorial del litoral de Galicia bajo un planteamiento integrado, y en la revisión del Plan Insular de Ordenación de Fuerteventura, donde se proponen fijar líneas estratégicas territoriales fruto de un mayor consenso.

Es del todo necesario que la planificación y la gestión pública deban partir de una visión transversal, dinámica e integradora, lo que justifica que le siga un capítulo dedicado a la gestión de espacios de destino turístico. En primer lugar, desde la escala local y sobre áreas frágiles, caso de los centros históricos, queda patente que la turistización de sus principales ejes urbanos no paraliza la gentrificación, como se ha demostrado en Salamanca; en cambio, en la isla de Pascua, en la que el auge del turismo ha generado un presión ambiental y demográfica, ha partido de la propia sociedad civil la creación de nuevas formas de gestión turística y control territorial. En este sentido, la gestión turística ostenta capacidad para fomentar el desarrollo sostenible, como se ha demostrado en la ciudad mexicana de Guanajuato en relación con el consumo de agua; o en aprovechar las herramientas de promoción turística para redistribuir flujos de visitantes en zonas saturadas, sin tener que renunciar a ellos.

En segundo lugar y a escala supramunicipal, la gestión pública mancomunada del turismo cobra éxito en la provincia de Barcelona, al ser pionera en nivel estatal en la incorporación de la dimensión territorial, expresada en el contacto constante con los agentes del territorio, y estructurando planes y programas en términos de territorio. En esta misma escala se estudian dos sistemas de gestión, el primero enfocado al patrimonio cultural de la eurorregión Galicia-Norte de Portugal, para mejorar su conocimiento y ampliar su difusión; y el segundo enmarcado en el desarrollo territorial del turismo y en la gestión sostenible de destinos, a través del diseño e implementación de un sistema de indicadores en destinos turísticos de la provincia de Barcelona. 
La sostenibilidad y los impactos que genera el turismo fundamentan el quinto capítulo de esta obra, exponiéndose, en primer lugar, los resultados del marco teórico de una investigación al objeto de desarrollar un sistema de indicadores aplicado a ciudades turísticas. En este mismo espacio, resulta de interés conocer diferentes aproximaciones metodológicas para determinar la huella turística aplicándose al caso de Madrid. Otro de los ámbitos territoriales que cobran funcionalidad turística son los espacios naturales protegidos, en ellos la sostenibilidad se analiza de la mano del uso de nuevas tecnologías con la finalidad de fundamentar una gestión inteligente. Finalmente, en áreas en declive, en Teruel, la implementación de estrategias de desarrollo territorial ha conseguido atenuar el progresivo deterioro socioeconómico. Por último, el estudio de los impactos derivados del desarrollo turístico, se focaliza por una parte en demostrar, en el destino de Guanajuato (México), que existe una relación positiva entre la concentración de contaminantes en el agua residual y la llegada de turistas al destino; y por otra, en las variables que condicionan el emplazamiento hotelero en el territorio a través del análisis clúster, categorizando los hoteles de Torremolinos.

La generación de experiencias a los visitantes precisa de la puesta en valor turístico de nuevos recursos y la creación de nuevas modalidades turísticas, dedicándose el último capítulo. Ello implica procesos de cambio en los destinos, con potencialidad para generar sinergias que revaloricen el territorio y generar beneficios a largo plazo, como es el caso del cineturismo en Sicilia; o en los espacios culturales de Copenhague, si bien pese a la protección de los recursos turísticos y las acciones de renovación urbana, no se ha generado ningún producto en torno a este patrimonio. Sin dejar de lado este patrimonio, se analiza la presencia de las mujeres trabajadoras textiles en el relato de espacios expositivos dedicados a colonias industriales de Catalunya. El desarrollo de nuevos destinos y sus transformaciones socioespaciales se expresa en espacios declarados monumentos nacionales, como es el caso de Villa Leyva (Colombia); o el hecho que para los turistas y viajeros espirituales lo religioso no juega un papel importante ante los recursos que conforman un paisaje sagrado en San Marcos de la Laguna (Guatemala). Por último, el paisaje, como recurso para reposicionar la oferta turística y fortalecer la identidad territorial, queda expresado en Asturias en los paisajes de la sidra y en la Vall de Camprodon a través de la Carta del Paisaje.

La obra expone a través de cuatro posters resultados de investigaciones, en las que se expresa como interrogante si la planificación turística genera cooperación en la gestión pública. Se demuestra, en el casco antiguo de Palma, la expansión de la frontera de gentrificación comercial derivada de la transformación funcional del retailscape provocado por la reestructuración turística. En esta línea de transformación funcional, se plasma la necesidad de gestionar un espacio natural protegido de forma activa, al objeto de evitar su transformación en parque temático, como se justifica en el Parque Natural de Somiedo; y en las áreas rurales de la Comunidad de Madrid el papel que desempeñan los agentes de empleo y desarrollo local en la planificación y gestión territorial del turismo.

En definitiva, el libro "De lugar geográfico a destino turístico. Análisis, planificación y gestión de los procesos de cambio generados por el turismo", es una obra de interés para académicos y profesionales de la planificación y gestión del turismo. Permite acceder a las 42 aportaciones presentadas en el XVI Coloquio de Geografía del Turismo, Ocio y Recreación, que reflejan la profusión de la investigación geográfica en turismo y asimismo desde disciplinas afines. Ello traduce el hecho de que un lugar 
es susceptible de convertirse en espacio de destino turístico e internacionalizarse, lo cual conlleva a esta apuesta multidisciplinar y a diversas escalas espaciales dada la transversalidad del turismo sobre el territorio.

Juan Bautista Ferreres Bonfill

Departamento de Historia, Geografía y Arte. Gabinete de Estudios Turísticos (GETUR)

Universitat Jaume I de Castellón 